\title{
Equivalence of the Self-Dual Model and Maxwell-Chern-Simons Theory on Arbitrary Manifolds
}

\author{
Emil M. Prodanovi and Siddhartha Sen \\ School of Mathematics, Trinity College, Dublin 2, Ireland, \\ e-mail: prodanov@maths.tcd.ie, sen@maths.tcd.ie
}

\begin{abstract}
Using a group-invariant version of the Faddeev-Popov method we explicitly obtain the partition functions of the Self-Dual Model and Maxwell-Chern-Simons theory. We show that their ratio coincides with the partition function of abelian Chern-Simons theory to within a phase factor depending on the geometrical properties of the manifold.
\end{abstract}

PACS numbers: 02.40.-k, 11.10.Kk, 11.15.-q, 11.90.+t

Keywords: Chern-Simons, Self-Dual Model, Faddeev-Popov, Ray-Singer, zeta-regularization, multiplicative anomaly.

*Supported by FORBAIRT scientific research program and Trinity College, Dublin. 


\section{Introduction}

In three dimensions it is possible to add a gauge-invariant Chern-Simons term to the Maxwell gauge field action [1], [2], [3]. The resulting Maxwell-ChernSimons theory has been analyzed completely and in 2] the entire subject of topologically massive three-dimensional gauge theories has been set up. Further Maxwell-Chern-Simons theory has been used as an effective theory for different models, such as fractional Hall effect and high-temperature superconductivity [4], [5].

The Self-Dual Model was first studied in detail by Deser et al. [2] and it was shown in [6] that the Self-Dual Model is equivalent, modulo global differences, to the Maxwell-Chern-Simons theory.

Subsequently, this equivalence has been studied by many authors using variety of techniques: in the context of bosonisation and at the quantum level (using Legendre transformation) in the abelian and non-abelian case in [7], [8]; by constraint analysis in [9] and [10]; by means of Batalin-FradkinTyutin formalism [11; in the context of duality [12], [13, [14 and many others.

We address the equivalence with differential geometric tools. It allows us to reveal global features of these models which, so far, have been overlooked. We pay particular attention to the zero modes present in the problem. These zero modes contain topological information regarding the manifold. By neglecting them, i.e. absorbing the divergence due to the zero modes in the normalization constant, this information is lost. A method, due to Schwarz [15], involving invariant integration, allows us to formally consider a key part of the zero mode sector from the divergent term. This is enough, as we show, to get topological information regarding the manifold.

We show that, subject to choice of appropriate renormalizations, the ratio of the partition functions of the two theories in the presence of currents is given, modulo phase factor, by the partition function of abelian ChernSimons theory with currents. This phase factor captures the geometrical properties of the manifold. The partition function of Chern-Simons theory contains a phase factor which captures the topological properties of the currents (their linking number) and modulo this phase factor it is a topological invariant (the Ray-Singer torsion of the manifold). Therefore the Self-Dual Model and Maxwell-Chern-Simons theory are equivalent to within a phase factor which contains geometrical information about the manifold and another phase factor which contains information about the topological properties of the currents. In our considerations we have used zeta-function regularised determinants which lead to phase ambiguities. 


\section{Review of Schwarz's group-invariant description of the Faddeev-Popov method}

In this section for the sake of completeness we will review, following [15], the method of reducing an integral of a function with some symmetries over some space to an integral over a lower-dimensional space.

Take $M$ to be a Riemannian manifold and $G$ - a compact group. Let $W=M / G$ denote the space of orbits.

Using the Riemannian metric, there are no problems in defining volume elements on $W$ and $M$.

Let $\lambda(x)$ be the volume of the orbit $G x$ with respect to the Riemannian metric on $M . \lambda(x)$ is $G$-invariant (since $\lambda(g x)=\lambda(x)$ for $g \in G$ ). Therefore $\lambda(x)$ is a function on $W=M / G$.

Let $f(x)$ be $G$-invariant function on $W=M / G$.

Hence:

$$
\int_{M} f(x) d \mu=\int_{M / G} f(x) \lambda(x) d v
$$

Define the linear operator $T_{x}: \operatorname{Lie}(G) \longrightarrow T_{x}(M)$, where $T_{x}(M)$ is the tangent space to $M$ at $x$ and $\operatorname{Lie}(G)$ is the Lie algebra of the group $G$.

Let $H_{x}$ be the stabilizer of the group $G$ at $x$, i.e.:

$$
H_{x} x=x
$$

Therefore:

$$
\operatorname{ker}\left(T_{x}\right)=\operatorname{Lie}\left(H_{x}\right)=\mathcal{H}_{x}
$$

Consider the linear operator $\widetilde{T_{x}}: \operatorname{Lie}(G) / \operatorname{Lie}\left(H_{x}\right) \quad \longrightarrow \quad T_{x}(M)$.

The operator $T_{x}^{\dagger} T_{x}$ is non-degenerate if, and only if, $G$ acts with discrete stabilizers. The operator $\widetilde{T_{x}^{\dagger}} \widetilde{T_{x}}$ is always non-degenerate. The quotient $G / H_{x}$ is homeomorphic to the orbit $G x$ under the map $g \mapsto g x$ for $g \in G$. The differential of this map at the identity coincides with the operator $\widetilde{T_{x}}$. Therefore:

$$
\operatorname{vol}(G x)=\operatorname{vol}\left(G / H_{x}\right)\left|\operatorname{det} \widetilde{T_{x}}\right|=\operatorname{vol}\left(G / H_{x}\right)\left(\operatorname{det} \widetilde{T_{x}^{\dagger}} \widetilde{T_{x}}\right)^{1 / 2}
$$

But

$$
\operatorname{vol}(G)=\int_{G} D g=\int_{G / H_{x}} D[g] \operatorname{vol}\left(H_{x}\right)=\operatorname{vol}\left(H_{x}\right) \operatorname{vol}\left(G / H_{x}\right)
$$

Take $\operatorname{vol}(G)$ to be normalized to 1 .

Then the volume of the orbit of the group is:

$$
\lambda(x)=\frac{1}{\operatorname{vol}\left(H_{x}\right)}\left(\operatorname{det} \widetilde{T_{x}^{\dagger}} \widetilde{T_{x}}\right)^{1 / 2}
$$


We now assume that all stabilizers are conjugate and have the same volume $\operatorname{vol}(H)$.

Then:

$$
\int_{M} f(x) d \mu=\frac{1}{\operatorname{vol}(H)} \int_{M / G} f(x)\left(\operatorname{det} \widetilde{T_{x}^{\dagger}} \widetilde{T_{x}}\right)^{1 / 2} d v
$$

With this formula we have restricted the gauge freedom by picking up only one representative from each orbit. Alternatively, we could have imposed a gauge-fixing condition and inserted it in the action together with the Faddeev-Popov determinant. This would bring a delta-function of the gaugefixing condition into the integrand and therefore would define a subspace in M. If this gauge-fixing condition is appropriate, this subspace would intersect each orbit exactly once and therefore the integration would pick up one representative of each orbit. For our further considerations (quadratic action functionals) we can also choose the resolvent method [16] — an invariant and beautiful form of the Faddeev-Popov trick.

\section{Partition Function of the Self-Dual Model}

The Self-Dual Model is given by the action:

$$
S_{S D}=\int_{M}\left(f_{\mu} f^{\mu}+\epsilon_{\mu \nu \lambda} f^{\mu} \partial^{\nu} f^{\lambda}\right) d^{3} x=\left\langle f,\left(\mathbb{I}+* d_{1}\right) f\right\rangle
$$

where $d_{p}$ is the map from the space of all $\mathrm{p}$-forms to the space of $(\mathrm{p}+1)$ forms, i.e. $d_{p}: \Omega^{p}(M) \longrightarrow \Omega^{p+1}(M)$, and $*$ is the Hodge star operator: * : $\Omega^{p}(M) \longrightarrow \Omega^{m-p} \quad(m=\operatorname{dim} M=3)$. The Hodge star operator explicitly depends on the metric of the manifold $M$.

Here we have introduced the notation of differential forms. Namely, we can write the scalar product of two p-forms $a_{p}$ and $b_{p}$ as: $\left\langle a_{p}, b_{p}\right\rangle=\int_{M} a_{p} \wedge * b_{p}$.

In this notation $\int_{M} f_{\mu} f^{\mu} d^{3} x$ may be written as $\int_{M} f \wedge * f$ and thus explicitly involves the metric and violates topological invariance. By "topological invariance" we will always mean metric-independence. The Chern-Simons term, in spite of the presence of $*$, is actually a topological invariant as it can be written simply as $\int_{M} A \wedge d A$ involving only differential forms which do not depend on the metric. The partition function of the model is:

$$
Z_{S D}=\frac{1}{N_{S D}} \int_{\Omega^{1}(M)} \mathcal{D} f e^{-i\left\langle f,\left(\mathbb{I}+* d_{1}\right) f\right\rangle}
$$


where $\Omega^{1}(M)$ is the space of all 1-forms on $M$ and $N_{S D}$ is a normalization factor.

The operator $\mathbb{I}+* d_{1}$ is self-adjoint.

Now we will extract the zero-mode dependence from the action functional. To do so, decompose $\Omega^{1}(M)$ :

$$
\Omega^{1}(M)=\operatorname{ker}\left(\mathbb{I}+* d_{1}\right) \oplus \operatorname{ker}\left(\mathbb{I}+* d_{1}\right)^{\perp}
$$

Therefore:

$$
Z_{S D}=\frac{1}{N_{S D}} \operatorname{vol}\left(k e r\left(\mathbb{I}+* d_{1}\right)\right) \operatorname{det}^{\prime}\left(i\left(\mathbb{I}+* d_{1}\right)\right)^{-1 / 2}
$$

Here we have retained, formally, the infinite volume factor. This can be removed by choosing the normalization constant $N_{S D}$ appropriately.

Witten has shown [17 how to deal with $i$ in $\operatorname{det}^{\prime}(i T)$ for some operator $T$ using $\zeta$-regularization technique. He found that $i$ leads to a phase factor, depending on the $\eta$-function of the operator $T$ and explicitly involving the metric of the manifold. For our case we have:

$$
\operatorname{det}^{\prime}\left(i\left(\mathbb{I}+* d_{1}\right)\right)^{-1 / 2}=e^{-\frac{i \pi}{4} \eta\left(0,\left(\mathbb{I}+* d_{1}\right)\right)} \operatorname{det}^{\prime}\left(\mathbb{I}+* d_{1}\right)^{-1 / 2}
$$

Finally, the partition function of the Self-Dual Model is:

$$
Z_{S D}=\frac{1}{N_{S D}} e^{-\frac{i \pi}{4} \eta\left(0,\left(\mathbb{I}+* d_{1}\right)\right)} \operatorname{vol}\left(k e r\left(\mathbb{I}+* d_{1}\right)\right) \operatorname{det}^{\prime}\left(\mathbb{I}+* d_{1}\right)^{-1 / 2}
$$

\section{Partition Function of Maxwell-Chern-Simons Theory}

The action of Maxwell-Chern-Simons Theory is:

$$
\begin{aligned}
S_{M C S} & =\int_{M}\left(F_{\mu \nu} F^{\mu \nu}+\epsilon_{\mu \nu \lambda} A^{\mu} \partial^{\nu} A^{\lambda}\right) d^{3} x \\
& =\left\langle A, d_{1}^{\dagger} d_{1} A\right\rangle+\left\langle A, * d_{1} A\right\rangle
\end{aligned}
$$

where $d_{k}^{\dagger}: \Omega^{k+1}(M) \longrightarrow \Omega^{k}(M)$.

In this case the topological invariance is explicitly violated by the Maxwell term.

On three-dimensional manifolds we have: $d_{1}^{\dagger}=* d_{1} *$. Therefore we may write the partition function as:

$$
Z_{M C S}=\frac{1}{N_{M C S}} \int_{\Omega^{1}(M)} \mathcal{D} A e^{-i\left\langle A,\left(* d_{1}+\left(* d_{1}\right)^{2}\right) A\right\rangle}
$$


The operator $* d_{1}+\left(* d_{1}\right)^{2}$ is self-adjoint.

We proceed to explicitly calculate the partition function. The theory has a gauge invariance under gauge transformations $A_{\mu} \longrightarrow A_{\mu}-\partial_{\mu} \lambda$, i.e.:

$$
A \longrightarrow A+d_{0} \Omega^{0}(M)
$$

We thus have an infinite-dimensional analogue of the situation reviewed in Section 2. To proceed we pick up one representative of each equivalence class $[A]$, where $[A]=A+d_{0} \Omega^{0}(M)$. To do this we impose the gauge condition $\partial_{\mu} A^{\mu}=0$, that is $d_{0}^{\dagger} A=0$.

This ensures that the space of orbits of the gauge group in the space of all 1-forms is orthogonal to the space of those $A$ 's, for which $d_{0}^{\dagger} A=0$ and so we will pick up only one representative of each orbit.

Then the operator $d_{0}$ plays the role of $\widetilde{T_{x}^{\dagger}}$ of Section 2, i.e. the stabilizer consists of those elements of $\Omega^{0}(M)$, for which $d_{0} \Omega^{0}(M)=0$ (the constant functions). Hence: $H=\mathbb{R}$.

Therefore:

$$
Z_{M C S}=\frac{1}{N_{M C S}} \frac{1}{\operatorname{vol}(H)} \int_{\Omega^{1}(M) / G} \mathcal{D} A e^{-i\left\langle A,\left(* d_{1}+\left(* d_{1}\right)^{2}\right) A\right\rangle}\left(\operatorname{det}^{\prime} d_{0}^{\dagger} d_{0}\right)^{1 / 2}
$$

The operator in the exponent has zero-modes.

Let $A \in \operatorname{ker}\left(* d_{1}+\left(* d_{1}\right)^{2}\right)$, i.e. $* d_{1} A+* d_{1} * d_{1} A=0$. There are two situations to consider. We can take $* d_{1} A=0$, that is: $A \in k e r\left(* d_{1}\right)$, or $A \notin k e r\left(* d_{1}\right)$, i.e. $* d_{1} A \neq 0$, but $* d_{1} A=-\left(* d_{1}\right)^{2} A$. In the second case $* d_{1}$ has inverse $\left(* d_{1}\right)^{-1}$. Therefore: $A=-* d_{1} A$, which means that $\left(\mathbb{I}+* d_{1}\right) A=0$, i.e. $A \in \operatorname{ker}\left(\mathbb{I}+* d_{1}\right)$.

By definition $k e r\left(* d_{1}\right) \cap \operatorname{ker}\left(\mathbb{1}+* d_{1}\right)=\emptyset$.

It is easy to see that $\operatorname{ker}\left(* d_{1}\right)$ and $\operatorname{ker}\left(\mathbb{I}+* d_{1}\right)$ are orthogonal:

Let $f \in \operatorname{ker}\left(\mathbb{I}+* d_{1}\right)$ and $g \in \operatorname{ker}\left(* d_{1}\right)$.

$\langle f, g\rangle=\langle f, g\rangle+\left\langle f, * d_{1} g\right\rangle=\left\langle f,\left(\mathbb{I}+* d_{1}\right) g\right\rangle=$

$=\left\langle\left(\mathbb{I}+* d_{1}\right) f, g\right\rangle=0$, since $\left(\mathbb{I}+* d_{1}\right)$ is self-adjoint and $f \in k e r\left(\mathbb{I}+* d_{1}\right)$.

So, $\operatorname{ker}\left(* d_{1}\right)$ is the orthogonal complement of $\operatorname{ker}\left(\mathbb{I}+* d_{1}\right)$.

Therefore we can write:

$$
\operatorname{ker}\left(* d_{1}+\left(* d_{1}\right)^{2}\right)=\operatorname{ker}\left(* d_{1}\right) \oplus \operatorname{ker}\left(\mathbb{I}+* d_{1}\right)
$$

The partition function is then given by:

$$
\begin{array}{r}
Z_{M C S}=\frac{1}{N_{M C S}} \frac{1}{\operatorname{vol}(H)} e^{-\frac{i \pi}{4} \eta\left(0, * d_{1}+\left(* d_{1}\right)^{2}\right)} \operatorname{vol}\left(\operatorname{ker}\left(* d_{1}+\left(* d_{1}\right)^{2}\right)\right) \\
\operatorname{det}^{\prime}\left(* d_{1}+\left(* d_{1}\right)^{2}\right)^{-1 / 2} \operatorname{det}^{\prime}\left(d_{0}^{\dagger} d_{0}\right)^{1 / 2}
\end{array}
$$


Because of (18) we can write this as:

$$
\begin{array}{r}
Z_{M C S}=\frac{1}{N_{M C S}} \frac{1}{\operatorname{vol}(H)} e^{-\frac{i \pi}{4} \eta\left(0, * d_{1}+\left(* d_{1}\right)^{2}\right)} \operatorname{vol}\left(k e r\left(* d_{1}\right)\right) \operatorname{vol}\left(k e r\left(\mathbb{I}+* d_{1}\right)\right) \\
\operatorname{det}^{\prime}\left(* d_{1}+\left(* d_{1}\right)^{2}\right)^{-1 / 2} \operatorname{det}^{\prime}\left(d_{0}^{\dagger} d_{0}\right)^{1 / 2}(2
\end{array}
$$

Let us now consider $\operatorname{det}^{\prime}\left(* d_{1}+\left(* d_{1}\right)^{2}\right)^{-1 / 2}$.

In the infinite-dimensional case we have to take into account the multiplicative anomaly, i.e. the fact that the determinant of a product of operators is not always the product of the determinants of the operators.

For our case we will show that:

$$
\operatorname{det}^{\prime}\left(* d_{1}+\left(* d_{1}\right)^{2}\right)=(-1)^{\psi} \operatorname{det}^{\prime}\left(* d_{1}\right) \operatorname{det}^{\prime}\left(\mathbb{I}+* d_{1}\right)
$$

where: $\psi=\zeta\left(0,-\left(* d_{1}+\left(* d_{1}\right)^{2}\right)_{-}\right)-\zeta\left(0,-\left(* d_{1}\right)_{-}\right)-\zeta\left(0,-\left(\mathbb{I}+* d_{1}\right)_{-}\right)$. The meaning of $\zeta\left(0, A_{-}\right)$will become clear from the context of the proof. Take $A$ to be some operator without zero-modes.

We can always write $A$ in the form: $A=\left(\begin{array}{cc}A_{+} & \\ & A_{-}\end{array}\right), \quad|A|=\left(\begin{array}{ll}A_{+} & \\ & -A_{-}\end{array}\right)$ where $A_{ \pm}: \quad \Gamma_{ \pm} \longrightarrow \Gamma_{ \pm}$and $\Gamma_{ \pm}$is the space spanned by eigenvectors of $A$ corresponding to positive (negative) eigenvalues. The operator $|A|$ has positive eigenvalues only. If we denote by $\lambda_{n}$ the eigenvalues of the operator $A$, for $\operatorname{det}|A|$ we can use the $\zeta$-regularised expresion to define:

$$
\operatorname{det}|A|=\prod_{n=1}^{\infty} \lambda_{n}=e^{-\zeta^{\prime}(0,|A|)}
$$

For any real number $\beta$ we have:

$$
\operatorname{det}(\beta|A|)=\beta^{-\zeta(0,|A|)} \operatorname{det}|A|
$$

The determinant of any operator $A$ can be written as:

$$
\operatorname{det}(A)=\operatorname{det}\left(A_{+}\right) \operatorname{det}\left(-\left(-A_{-}\right)\right)=(-1)^{-\zeta\left(0,-A_{-}\right)} \operatorname{det}|A|
$$

Let $A=* d_{1}, \quad B=\mathbb{I}+* d_{1}$.

For the determinants we write the formal expressions which are always to be regularised using (22) as:

$$
\operatorname{det}|A|=\prod_{n=1}^{\infty}\left|\lambda_{n}\right|, \quad \operatorname{det}|B|=\prod_{n=1}^{\infty}\left|1+\lambda_{n}\right|
$$


Denote by $F(U, V)=\frac{\operatorname{det}(U V)}{\operatorname{det}(U) \operatorname{det}(V)}$ the multiplicative anomaly. In general $F(U, V) \neq 1$, as well as: $\ln \operatorname{det}(U) \neq \operatorname{tr} \ln (U)$.

We have:

$$
\ln F(|A|,|B|)=-\frac{d}{d s}(\zeta(s,|A B|)-\zeta(s,|A|)-\zeta(s,|B|))_{s=0}=0
$$

$\zeta(s,|U|)$ can be expressed as:

$$
\zeta(s,|U|)=\zeta\left(s, U_{+}\right)+\zeta\left(s,-U_{-}\right)
$$

Therefore:

$$
\begin{aligned}
\ln F(|A|,|B|)=- & \frac{d}{d s}\left(\zeta\left(s,(A B)_{+}\right)+\zeta\left(s,(A B)_{-}\right)-\right. \\
& \left.-\zeta\left(s, A_{+}\right)-\zeta\left(s, A_{-}\right)-\zeta\left(s, B_{+}\right)-\zeta\left(s, B_{-}\right)\right)_{s=0}
\end{aligned}
$$

For all operators entering (28) we can apply the analysis of [18]. This analysis holds for the case of a smooth and compact manifold. The Seeley-De Witt formula:

$$
\zeta(s, U)=\frac{1}{\Gamma(s)}\left(\sum_{n=0}^{\infty} \frac{A_{n}}{s+n-\frac{D}{2}}+J(s)\right)
$$

where $A_{n}$ are the heat-kernel coefficients, $D$ is the dimension of the manifold and $J(s)$ is some analytic function, leads to the fact that the multiplicative anomaly will vanish when $D=2$ or $D$ is odd. Therefore:

$$
\operatorname{det}^{\prime}\left(* d_{1}+\left(* d_{1}\right)^{2}\right)=e^{i \pi \psi} \operatorname{det}^{\prime}\left(* d_{1}\right) \operatorname{det}^{\prime}\left(\mathbb{I}+* d_{1}\right)
$$

where: $\quad \psi=\zeta\left(0,-\left(* d_{1}+\left(* d_{1}\right)^{2}\right)_{-}\right)-\zeta\left(0,-\left(* d_{1}\right)_{-}\right)-\zeta\left(0,-\left(\mathbb{I}+* d_{1}\right)_{-}\right)$. Note that the appearance of the phase factor is not due to the multiplicative anomaly. We have used the fact that the multiplicative anomaly vanishes for the moduli of the operators. However, from (24) we see that we are forced to include some phase ambiguity which is related to the "negative" parts of the operators - otherwise we would not be able to define a zeta-function regularized expressions for operators which have negative eigenvalues].

It follows that the partition function of Maxwell-Chern-Simons theory can be written as:

$$
\begin{gathered}
Z_{M C S}=\frac{e^{-\frac{i \pi}{2} \psi-\frac{i \pi}{4} \eta\left(0, * d_{1}+\left(* d_{1}\right)^{2}\right)}}{N_{M C S}} \frac{1}{\operatorname{vol}(H)} \operatorname{vol}\left(k e r\left(* d_{1}\right)\right) \operatorname{vol}\left(k e r\left(\mathbb{I}+* d_{1}\right)\right) \\
\operatorname{det}^{\prime}\left(* d_{1}\right)^{-1 / 2} \operatorname{det}^{\prime}\left(\mathbb{I}+* d_{1}\right)^{-1 / 2} \operatorname{det}^{\prime}\left(d_{0}^{\dagger} d_{0}\right)^{1 / 2}
\end{gathered}
$$

\footnotetext{
${ }^{1}$ For other examples of phase ambiguities associated with $\zeta$-regularised determinants see for instance [19 and 20.
} 
The $*$-operator is invertible, hence: $\operatorname{ker}\left(* d_{1}\right)=\operatorname{ker}\left(d_{1}\right)$.

We proceed to write (31) in a more convenient form in which topological features are highlighted. We note that the projection map $k e r\left(d_{k}\right) \longrightarrow H_{d R}^{q}(M)$ induces the isomorphism:

$$
\phi_{q}: \mathcal{H}^{q}(M) \longrightarrow H_{d R}^{q}(M),
$$

where $H_{d R}^{q}(M)$ is the $q^{\text {th }}$ deRham cohomology group and $\mathcal{H}^{q}(M)$ is the space of the harmonic q-forms. Therefore:

$$
\operatorname{vol}\left(k e r\left(d_{q}\right)\right)=\left|\operatorname{det} \phi_{q}\right|^{-1} \operatorname{vol}\left(H_{d R}^{q}(M)\right) \text {. }
$$

All inner products are in the space of the harmonic forms. In the deRham cohomology group there is no *-operator - it is purely topological. The reason for introducing the deRham cohomology groups is that we would like to isolate the metric dependence which is present in the volume elements and in the inner products.

The stabilizer $H$ consists of those elements of $\Omega^{0}(M)$ for which $d_{0} \Omega^{0}=0$, i.e. $H=\operatorname{ker}\left(d_{0}\right)$. So the volume of the stabilizer is:

$$
\operatorname{vol}(H)=\operatorname{vol}\left(H_{d R}^{0}(M)\right)\left|\operatorname{det} \phi_{0}\right|^{-1} .
$$

Finally, the partition function of Maxwell-Chern-Simons theory is:

$$
\begin{gathered}
Z_{M C S}=\frac{e^{-\frac{i \pi}{2} \psi-\frac{i \pi}{4} \eta\left(0, * d_{1}+\left(* d_{1}\right)^{2}\right)}}{N_{M C S}} \frac{\operatorname{vol}\left(H_{d R}^{1}(M)\right)}{\operatorname{vol}\left(H_{d R}^{0}(M)\right)} \frac{\operatorname{det}\left(\phi_{0}^{\dagger} \phi_{0}\right)^{1 / 2}}{\operatorname{det}\left(\phi_{1}^{\dagger} \phi_{1}\right)^{1 / 2}} \operatorname{det}^{\prime}\left(d_{0}^{\dagger} d_{0}\right)^{1 / 2} \\
\operatorname{det}^{\prime}\left(* d_{1}\right)^{-1 / 2} \operatorname{det}^{\prime}\left(\mathbb{I}+* d_{1}\right)^{-1 / 2} \operatorname{vol}\left(k e r\left(\mathbb{I}+* d_{1}\right)\right)
\end{gathered}
$$

The volume elements in (35) are not finite. We can define the normalization constant to be the inverse of the ratio of the volumes of the deRham cohomology groups. Using this fact and (13), (20), (30), and (33) we get:

$$
\begin{aligned}
Z_{M C S}=e^{-\frac{i \pi}{2} \psi-\frac{i \pi}{4} \eta\left(0, * d_{1}+\left(* d_{1}\right)^{2}\right)+\frac{i \pi}{4} \eta\left(0, \mathbb{I}+* d_{1}\right)} \\
\frac{\operatorname{det}\left(\phi_{0}^{\dagger} \phi_{0}\right)^{1 / 2}}{\operatorname{det}\left(\phi_{1}^{\dagger} \phi_{1}\right)^{1 / 2}} \operatorname{det}^{\prime}\left(d_{0}^{\dagger} d_{0}\right)^{1 / 2} \operatorname{det}^{\prime}\left(d_{1}^{\dagger} d_{1}\right)^{-1 / 4} Z_{S D}
\end{aligned}
$$

We now note that expression (36) for the ratio of the partition functions of Maxwell-Chern-Simons theory and the Self-Dual Model is equal, modulo phase factor, to the partition function of pure abelian Chern-Simons theory円. Thus:

$$
\frac{Z_{M C S}}{Z_{S D}}=e^{i \alpha} Z_{C S}
$$

\footnotetext{
${ }^{1}$ This relationship, without the phase factor, was established in [9] and 14 by different analyses. We would like to thank P. J. Arias and J. Stephany for drawing our attention to these references.
} 
where

$$
\begin{aligned}
\alpha=-\frac{\pi}{2} \zeta(0, & \left.-\left(* d_{1}+\left(* d_{1}\right)^{2}\right)_{-}\right)+\frac{\pi}{2} \zeta\left(0,-\left(* d_{1}\right)_{-}\right)+\frac{\pi}{2} \zeta\left(0,-\left(\mathbb{I}+* d_{1}\right)_{-}\right) \\
& +\frac{\pi}{4} \eta\left(0, * d_{1}\right)+\frac{\pi}{4} \eta\left(0, \mathbb{I}+* d_{1}\right)-\frac{\pi}{4} \eta\left(0, * d_{1}+\left(* d_{1}\right)^{2}\right)
\end{aligned}
$$

The partition function of abelian Chern-Simons theory [21], 22] is equal, modulo phase factor, to the square root of the Ray-Singer torsion which is a topological invariant of the manifold given by [21]:

$$
\tau_{R S}(M)=\prod_{q=0}^{3}\left(\left|\operatorname{det} \phi_{q}\right|\left|\operatorname{det}^{\prime} d_{q}\right|\right)^{(-1)^{q}}
$$

So the absolute value of ratio of the partition functions of Maxwell-ChernSimons theory and the Self-Dual Model is independent of the metric of the manifold and consequently these two theories are equivalent to within a phase factor on arbitrary manifolds.

Consider now the partition function of Maxwell-Chern-Simons theory with an external source $J$ coupled to the fields $A$ :

$$
Z_{M C S}(J)=\frac{1}{N_{M C S}} \int_{\Omega^{1}(M)} \mathcal{D} A e^{-i\left\langle A,\left(* d_{1}+\left(* d_{1}\right)^{2}\right) A\right\rangle+\langle J, A\rangle}
$$

For consistency we require that:

$$
J \in \operatorname{ker}\left(* d_{1}+\left(* d_{1}\right)^{2}\right)^{\perp}
$$

Decompose $\Omega^{1}(M)$ :

$$
\Omega^{1}(M)=k e r\left(* d_{1}+\left(* d_{1}\right)^{2}\right) \oplus \operatorname{ker}\left(* d_{1}+\left(* d_{1}\right)^{2}\right)^{\perp}
$$

Using (18) we may write:

$$
\begin{gathered}
Z_{M C S}(J)=\frac{1}{N_{M C S}} \operatorname{vol}\left(\operatorname{ker}\left(* d_{1}\right)\right) \operatorname{vol}\left(\operatorname{ker}\left(\mathbb{I}+* d_{1}\right)\right) \\
\int \mathcal{D} A e^{-i\left\langle A, * d_{1}\left(\mathbb{I}+* d_{1}\right) A\right\rangle+\langle J, A\rangle} \\
\operatorname{ker}\left(* d_{1}+\left(* d_{1}\right)^{2}\right)^{\perp}
\end{gathered}
$$

The integral gives:

$$
\begin{aligned}
& \int \mathcal{D} A e^{-i\left\langle A, * d_{1}\left(\mathbb{I}+* d_{1}\right) A\right\rangle+\langle J, A\rangle}= \\
& \operatorname{ker}\left(* d_{1}+\left(* d_{1}\right)^{2}\right)^{\perp} \\
& =\operatorname{det}^{\prime}\left(i\left(* d_{1}+\left(* d_{1}\right)^{2}\right)\right)^{-1 / 2} e^{i\left\langle J, \frac{1}{* d_{1}+\left(* d_{1}\right)^{2}} J\right\rangle}
\end{aligned}
$$


Using (30) we obtain:

$$
\begin{gathered}
Z_{M C S}(J)=\frac{e^{-\frac{i \pi}{2} \psi-\frac{i \pi}{4} \eta\left(0, * d_{1}+\left(* d_{1}\right)^{2}\right)}}{N_{M C S}} \operatorname{vol}\left(k e r\left(* d_{1}\right)\right) \operatorname{vol}\left(k e r\left(\mathbb{I}+* d_{1}\right)\right) \\
\operatorname{det}^{\prime}\left(* d_{1}\right)^{-1 / 2} \operatorname{det}^{\prime}\left(\mathbb{I}+* d_{1}\right)^{-1 / 2} e^{i\left\langle J, \frac{1}{* d_{1}+\left(* d_{1}\right)^{2}} J\right\rangle}
\end{gathered}
$$

Here we identify the Ray-Singer torsion [23]. Namely, with suitable choice of normalization $N$ :

$$
\frac{1}{N} \operatorname{vol}\left(k e r\left(* d_{1}\right)\right) \operatorname{det}^{\prime}\left(* d_{1}\right)^{-1 / 2}=\tau_{R S}(M)^{1 / 2}
$$

Hence:

$$
\begin{array}{r}
Z_{M C S}(J)=\frac{e^{-\frac{i \pi}{2} \psi-\frac{i \pi}{4} \eta\left(0, * d_{1}+\left(* d_{1}\right)^{2}\right)}}{N_{M C S}} \operatorname{det}^{\prime}\left(\mathbb{I}+* d_{1}\right)^{-1 / 2} \operatorname{vol}\left(k e r\left(\mathbb{I}+* d_{1}\right)\right) \\
\tau_{R S}(M)^{1 / 2} e^{i\left\langle J, \frac{1}{* d_{1}+\left(* d_{1}\right)^{2}} J\right\rangle}
\end{array}
$$

The determinant entering this expression can be written as:

$$
\begin{aligned}
\operatorname{det}^{\prime}\left(\mathbb{I}+* d_{1}\right)^{-1 / 2} & =e^{\frac{i \pi}{4} \eta\left(0, \mathbb{I}+* d_{1}\right)} \operatorname{det}^{\prime}\left(-i\left(\mathbb{I}+* d_{1}\right)\right)^{-1 / 2} \\
=e^{\frac{i \pi}{4} \eta\left(0, \mathbb{I}+* d_{1}\right)} e^{i\left\langle J, \frac{1}{\mathbb{I}+* d_{1}} J\right\rangle} \int \mathcal{D} A e^{-i\left\langle A,-\left(\mathbb{I}+* d_{1}\right) A\right\rangle+\langle J, A\rangle} & \int_{\operatorname{ker}\left(\mathbb{I}+* d_{1}\right)^{\perp}}
\end{aligned}
$$

In the integral we now change the variables from $A$ to $i A$. The Jacobian of this change of variables is $\operatorname{det}^{\prime}(i \mathbb{I})$ which is a constant and we can absorb it in the normalization factor.

The product of this determinant with the volume element gives (modulo normalization factor) the partition function of the Self-Dual Model with current $\hat{J}=-i J$. Therefore:

$$
Z_{M C S}(J)=e^{i \alpha-\frac{i \pi}{4} \eta\left(0, * d_{1}\right)} \tau_{R S}(M)^{1 / 2} e^{i\left\langle J, \frac{1}{* d_{1}} J\right\rangle} Z_{S D}(\hat{J})
$$

The first exponent contains the geometrical information of the manifold via the $\eta$-function, while the second one yields the linking number of the currents. The partition function of pure abelian Chern-Simons theory [23] in the presence of a current $J$ is

$$
Z_{C S}(J)=e^{-\frac{i \pi}{4} \eta\left(0, * d_{1}\right)} \tau_{R S}(M)^{1 / 2} e^{i\left\langle J, \frac{1}{* d_{1}} J\right\rangle}
$$

Therefore, at the level of currents, the ratio of the partition functions of Maxwell-Chern-Simons theory and Self-Dual Model is a topological invariant to within a phase factor:

$$
\frac{Z_{M C S}(J)}{Z_{S D}(\hat{J})}=e^{i \alpha} \tau_{R S}(M)^{1 / 2} e^{i\left\langle J, \frac{1}{* d_{1}} J\right\rangle}=e^{i \alpha} Z_{C S}(J)
$$


where:

$$
\begin{gathered}
\alpha=-\frac{\pi}{2} \zeta\left(0,-\left(* d_{1}+\left(* d_{1}\right)^{2}\right)_{-}\right)+\frac{\pi}{2} \zeta\left(0,-\left(* d_{1}\right)_{-}\right)+\frac{\pi}{2} \zeta\left(0,-\left(\mathbb{I}+* d_{1}\right)_{-}\right) \\
-\frac{\pi}{4} \eta\left(0, * d_{1}+\left(* d_{1}\right)^{2}\right)+\frac{\pi}{4} \eta\left(0, * d_{1}\right)+\frac{\pi}{4} \eta\left(0, \mathbb{I}+* d_{1}\right)
\end{gathered}
$$

The factor $\alpha$ contains the geometrical information of the manifold.

Note that the correlation functions can be calculated in the usual way by functionally differentiating the partition functions with respect to the external current. Equation (51) allows us to relate the correlation functions between the models.

As an example, let us take the manifold to be $S^{3}$ (hence the Ray-Singer torsion is 1 [24]) and let us suppose that the currents do not link. Then we get $Z_{C S}(J)=1$ and therefore Maxwell-Chern-Simons theory is equivalent to the Self-Dual Model to within a phase factor which captures the geometrical properties of the manifold. If the currents link then the partition functions differ by an additional phase which captures the topological features of the currents.

The main differences between our results and those of earlier authors can be summarized as follows. We consider arbitrary manifolds and show, for the complete theories, the surprising result that the ratio of these two theories is itself a complete topological field theory (i.e. Chern-Simons theory). We also note that when the manifold is $\mathbb{R}^{3}\left(S^{3}\right)$ and, as considered by earlier authors, with no topological entanglement of currents, then the partition function of the Chern-Simons theory is 1. This result is in exact agreement with the results obtained by earlier authors.

\section{Acknowledgements}

E. P. would like to thank David Adams for valuable discussions and Fabian Sievers for $\mathrm{HT}_{\mathrm{E}} \mathrm{X}$ tips. 


\section{References}

[1] R. Jackiw, S. Templeton, Phys. Rev. D23, 2291, (1981)

[2] S. Deser, R. Jackiw, and S. Templeton, Phys. Rev. Lett. 48, 975, (1982);

S. Deser, R. Jackiw, and S. Templeton, Ann. Phys. (N.Y.) 140, 372, (1982)

[3] J. Schonfeld, Nucl. Phys. B185, 157, (1981)

[4] F. Wilczek, Fractional Statistics and Anyon Superconductivity, World Scientific, 1990.

[5] A. P. Balachandran, L. Chandar, E. Ercolessi, T. R. Govindarajan, and R. Shankar, Int. J. Mod. Phys. A8, 3417, (1994)

[6] S. Deser, R. Jackiw, Phys. Lett. 139B, 371, (1984)

[7] E. Fradkin and F. A. Schaposnik, Phys. Lett. 338B, 253, (1994)

[8] N. Bralic, E. Fradkin, V. Manias and F. A. Schaposnik, Nucl. Phys. B446, 144, (1995)

[9] P. J. Arias and A. Restuccia, Phys. Lett. B347, 241, (1995)

[10] R. Banerjee, H. J. Rothe, K. D. Rothe, Phys. Rev. D52,3750, (1995)

[11] R. Banerjee, H. J. Rothe, Nucl. Phys. B447, 183, (1995)

[12] Y. I. Kogan, , Mod. Phys. Lett. A6, 501, (1991)

[13] J. C. Le Guillou, E. F. Moreno, C. Nunez and F. A. Schaposnik, Mod. Phys. Lett. A12, 2707, (1997)

[14] J. Stephany, Phys. Lett. B390, 128, (1997)

[15] A. S. Schwarz, Quantum Field Theory and Topology, SpringerVerlag, Berlin 1993

[16] A. S. Schwarz, Commun. Math. Phys. 64, 233-268, (1979)

[17] E. Witten, Commun. Math. Phys. 121, 351, (1989)

[18] E. Elizalde, L. Vanzo, S. Zerbini, Commun. Math. Phys. 194, 613, (1998)

[19] D. Adams and S. Sen, Phys. Lett. B353, 495, (1995) 
[20] S. Deser, L. Griguolo, and D. Seminara, Phys. Rev. D57, 7444, (1998)

[21] D. H. Adams, R-torsion and Linking Numbers from Simplicial Abelian Gauge Theories, hep-th/9612009

[22] A. S. Schwarz, Lett. Math. Phys. 2, 247, (1978)

[23] A. S. Schwarz, Yu. Tyupkin, Nucl. Phys. B242, 436, (1984)

[24] D. B. Ray, I. M. Singer, Adv. Math. 7, 145, (1971) 